\title{
Emotion recognition using fiducial points via deep learning
}

\author{
Usmain Ahmed, Jawad Khan
}

\section{INTRODUCTION}

Emotion awareness is critical because of the function that emotions play in our daily lives. As a result, automatic emotion recognition aims to provide a machine with the human ability to interpret and comprehend a person's emotional state in order to predict his intents based on his facial expression. In this research, a new method for improving the accuracy of emotion recognition from facial expression is proposed, which is based solely on input attributes deduced from fiducial points. First, 1200 dynamic features representing the percentage of euclidean distances between facial fiducial points in the first frame and facial fiducial points in the last frame are extracted from image sequences. Second, just the most relevant features are chosen using active learning method. Finally, to categorise facial expression input into emotion, the selected features are provided to a ResNet classifier.

\section{REFERENCES}

[1] Arca, S., Campadelli, P. and Lanzarotti, R., 2006. A face recognition system based on automatically determined facial fiducial points. Pattern recognition, 39(3), pp.432-443.

[2] Martínez, A., Alcaraz, R. and Rieta, J.J., 2010. Application of the phasor transform for automatic delineation of single-lead ECG fiducial points. Physiological measurement, 31(11), p.1467.

[3] Gowda, S.N., 2016, December. Age estimation by LS-SVM regression on facial images. In International Symposium on Visual Computing (pp. 370-379). Springer, Cham.

[4] Yin, L. and Basu, A., 1997, October. MPEG4 face modeling using fiducial points. In Proceedings of International Conference on Image Processing (Vol. 1, pp. 109-112). IEEE.

[5] Plesnik, E., Malgina, O., Tasič, J.F. and Zajc, M., 2012. Detection of the electrocardiogram fiducial points in the phase space using the euclidian distance measure. Medical engineering \& physics, 34(4), pp.524-529.

[6] Gowda, S.N., 2016, November. Fiducial points detection of a face using RBF-SVM and adaboost classification. In Asian Conference on Computer Vision (pp. 590-598). Springer, Cham.

[7] Campadelli, P. and Lanzarotti, R., 2002. Localization of facial features and fiducial points. In Processings of the International Conference Visualisation, Imaging and image Processing (VIIP2002), Malaga (Spagna) (pp. 491-495).

[8] Gowda, S.N. and Yuan, C., 2018, December. ColorNet: Investigating the importance of color spaces for image classification. In Asian Conference on Computer Vision (pp. 581-596). Springer, Cham.

[9] Y. Du, W. Wang, and L. Wang. Hierarchical recurrent neural network for skeleton based action recognition. In Proceedings of the IEEE conference on computer vision and pattern recognition, pages 1110-1118, 2015.

[10] X. Guo, J. Liu, and Y. Chen. Fitcoach: Virtual fitness coach empowered by wearable mobile devices. In IEEE INFOCOM 2017-IEEE Conference on Computer Communications, pages 1-9. IEEE, 2017.

[11] I. Habibie, W. Xu, D. Mehta, G. Pons-Moll, and C. Theobalt. In the wild human pose estimation using explicit $2 d$ features and intermediate $3 d$ representations. In Proceedings of the IEEE Conference on Computer Vision and Pattern Recognition, pages 10905-10914, 2019.

[12] Gowda, S.N., 2016, October. Face verification across age progression using facial feature extraction. In 2016 International Conference on Signal and Information Processing (IConSIP) (pp. 1-5). IEEE. 